\title{
Estimation of serum 25-hydroxy vitamin D and zinc levels in patients with acne vulgaris and their association with disease severity
}

Asmaa Awni Haydar ${ }^{1} *$ Nazanin Sadq Ismail ${ }^{2}$ Isra Mohammed Taha Alsaadi ${ }^{2}$ Marwa Miqdad Tahir ${ }^{1}$ Azgar Nash Hussen $^{1}$ Balen Nariman Tahir ${ }^{1}$ Mustafa Wrya Ikram ${ }^{1}$ Aveen Nozad Adham ${ }^{3}$ Lana Yousif Mutalib ${ }^{3}$ Bashdar M. Hussen $^{3}$

\section{Abstract}

Backgrounds and objective: Acne vulgaris is a common inflammatory skin disease. Vitamin D3 plays an important role in the immune system. Zinc also is an essential element for human which plays a vital role in the immune system and their deficient subjects may experience increased susceptibility to a variety of pathogens. Our study was done to determine the effect of vitamin $\mathrm{D}$ and zinc level deficiency on the development of acne.

Methods: This study was performed at Hawler Medical University/Medical Research Center and DNA laboratory, Erbil, Iraq, from August 2018 to September 2019.Thirty three samples were collected from acne patients of both genders participated in the study and twenty five samples were collected from subjects (with supplement treatment) as control group. Serum levels of $25(\mathrm{OH})$ D and zinc were determined by mini VIDAS $₫$ from bioMérieux.

Results: There were statistical significant differences in age (20.52year versus 35.52 year, respectively, and body mass index between Group I (Control) and Group II (Patients) $\left(23.55 \mathrm{~kg} / \mathrm{m}^{2}\right.$ versus $\left.26.74 \mathrm{~kg} / \mathrm{m}^{2}\right)$, respectively. A significant high value of vitamin D levels $(P \leq 0.001)$ and non-significant high frequency of zinc levels in acne vulgaris patients when compared with patients treated with supplements $(P \leq 0.248)$.

Conclusion: We found that vitamin $D$ deficiency was more frequent in patients with acne, which was inversely related with disease severity. A further study with a larger sample size is needed to confirm our results because of the small number of patients in the supplementation study and the natural fluctuation of acne.

Keywords: Acne vulgaris, Serum $25(\mathrm{OH})$ vitamin D, Zinc.

\section{Introduction}

Acne vulgaris is a chronic form of skin disease that involves sebaceous follicles which is a common basis of face, chest and back. Androgenic changes in serum sexual hormones which stimulate of sebum production by the adrenal glands of both males as well as females. The disease commonly occurs during with adolescents, however would also children also would be affected. ${ }^{1,2}$ Acne is one of the most common skin disorders worldwide and occurs primarily at puberty with a prevalence of almost 95\%. Although it is principally a disorder of adolescence, the prevalence of adult patients with acne, especially adult women with acne, is elevated. The higher prevalence of adult acne in the female population prompted us to investigate whether other variables could present significant differences between gender. ${ }^{3}$ Adult female acne affects around $40 \%$ of women and the prevalence of the disease in adult women is rising. Adult female acne is considered to be different from adolescent acne both in terms of its clinical presentation and pathogenesis. ${ }^{4} \mathrm{~A}$ combination of increased sebum formation and abnormal hyper proliferation of keratinocytes results in the formation of a small microscopic lesion known as microcomedo. ${ }^{5}$ In addition,

${ }^{1}$ Department of Pharmacology, College of Pharmacy, Hawler Medical University, Erbil, Iraq.

2 Ministry of Health, Erbil, Iraq.

${ }^{3}$ Department of Pharmacognosy, College of Pharmacy, Hawler Medical University, Erbil, Iraq.

* Correspondence: Asmaa.Haydar@hmu.edu.krd 
Propionibacterium acnes ( $P$. acnes) the main predisposing factor in acne vulgaris which is triggers cytokine activation by Toll-like receptors, which means that the innate immune system is also important for acne development. ${ }^{6}$ Many conditions which exacerbate the disease have been suggested including diet, menstruation, sweating, personal stress, ultraviolet radiation, application of pomades and occupation. Use of medications like lithium, steroids, and anticonvulsants, exposure to excess sunlight, use of occlusive wear like shoulder pads, headbands backpacks, and underwire brassieres, endocrine disorders like polycystic ovarian syndroms. ${ }^{7}$ Food with a high glycemic index is rapidly absorbed, increases serum glucose levels and stimulates increased glucosedependent insulin signaling. ${ }^{8}$ Apart from the named consequences, depression and eating complaints are the most prominent problems, which expose patients who are the risk of mental and dietary factors of severe and resistant disease. Concerning dietary factors, studies have been conducted to show the role of zinc in treatment of drug-resistant cases of depression. Also these kinds of performances have done for vitamin $D$ and its effects on acne vulgaris. ${ }^{9}$ Vitamin D as an antioxidant agent would have positive impression on the disease. ${ }^{10,11}$ At the basal layer of epidermis, the cholesterol is converted to pro- vitamin D3 (7-dehydrocholesterol), which undergoes a photochemical transformation due to ultraviolet $B$ radiation (UVB) into the intermediate compound pre- vitamin D3. ${ }^{12}$ Cholecalciferol synthesized in the skin is transferred to a circulatory system. The first activation is made in hepatic cells into 25-hydroxychole- calciferol - calcidiol [25 $(\mathrm{OH}) \mathrm{D}]$, which is the main form of vitamin circulating in the blood. The second stage occurs in the kidney cells with production of $1(\mathrm{OH}) 25$-di- hydroxycholecalciferol calcitriol $[1,25(\mathrm{OH}) \mathrm{D}]$, which is the main active form of the vitamin and a specific ligand for a nuclear vitamin $D$ receptor
(VDR). ${ }^{13}$ However, it is confirmed that the vitamin $D$ via its anti-apoptotic effect protects the epidermal melanin and melanocyte through a control over the activation, proliferation, migration of melanocytes and melanogenesis by modulating $T$ cell activation. The exact mechanism of these effects is not fully understood. Vitamin D probably exerts melanocyte physiology through melanogenic cytokines (such as endothelin -3) and regulatory factors of melanocyte life and maturation process. ${ }^{14}$ Zinc is a trace element found in the structure of many metalloenzymes that play a role in important functions such as protein synthesis, DNA and RNA replication and cell division. Zinc is therefore required for growth and development. ${ }^{15}$ Also zinc plays a vital role in the development of metabolic syndrome, taking part in the regulation of cytokine expression, suppressing inflammation, and is also required to activate antioxidant enzymes that scavenge reactive oxygen species, decreasing oxidative stress. Zinc is found to stimulate production of IL-2 and induces a shift from Th2 to Th1 response. It has also been illustrated to decrease the serum levels of TNF- $\alpha$ and inhibit the TNF- $\alpha$ induced apoptosis of peripheral blood mononuclear cells that helps in controlling the disease activity and reactional states. ${ }^{16}$ Zinc also have a role in the correct functioning of lipid and glucose metabolism, regulating and forming the expression of insulin. ${ }^{17}$ It has also been demonstrated to possess anti-androgenic properties as it causes modulation of $5 \alpha$-reductase type 1 and 2 activity. ${ }^{18,19}$ This study headed to compare serum levels of $25(\mathrm{OH})$ vitamin $D$ between cases of acne vulgaris and age- plus sex-matched of supplemental control group to find any relationship between the vitamin and the occurrence of the disease besides comparing the two groups in terms of vitamin and zinc level deficiency conditions. The aims of the present study to determine the effect of vitamin $D$ and 
zinc level deficiency on the development of acne in comparison with treatment group.

\section{Methods}

A prospective case-control study of acne vulgaris was performed at Hawler Medical University, College of Pharmacy in cooperation with acne vulgaris patients who referred to dermatology departments and clinics at Awat Center and DNA laboratory/Erbil//raq. The patients were recruited from the Dermatology department at Awat consultant center from April to November 2019. Eligible patients of both gender whatever age with a history of Acne vulgaris. Exclusion criteria prohibited enrollment of patients and controls who were receiving therapeutic interventions such as systemic hormonal therapy, pregnant women, severe illness, infants, elderly, a vitamin D and zinc supplements (group I). History and examination were carried out severity of acne was recorded using the Global Acne Grading System (GAGS). This system divides the face, chest and back into six areas (forehead, left cheek, right cheek, nose, chin and chest and back) and assigns a factor to each area on the basis of severity. A total number of 33 patients with acne vulgaris and 25 as control (with supplements 5000 IU vitamin D, Zinc gluconate $50 \mathrm{mg}$; daily oral dose for 3 months). Information on the whole population was recorded including their gender and age group. Anthropometric measurements including the height $(\mathrm{m})$, weight $(\mathrm{kg})$ and the calculated body mass index $\mathrm{kg} / \mathrm{m} 2$ were determined. A venous blood sample drew from each patient's serum test tube (for determination of vitamin D and zinc levels), and plain test tube to separate the serum by centrifugation at $3000 \mathrm{rpm}$ for 15 minutes to determine the inflammatory markers, they were determined by using enzyme linked immune sorbent assay technology according to the instruction of the manufacturer of mini VIDAS ${ }^{\circledR}$ from bioMérieux.

\section{Statistical Analysis}

The statistical result were computed by SPSS version 24 where many statistical tests were used to answer the objectives of the study. For example, the Chi-sqaure test was conducted to discover the association between the categorical variables and $P$ values were also discussed given in Table (3-1), also for changes in both measurements for all categorical variables were considered and tested using Kruskal Tests in case of more than two groups followed by Man-Whitney $U$ test for multiple comparison as mentioned in Table 2.

\section{Results}

The current study was the first, to our knowledge, in the evaluation of serum concentration of " $25(\mathrm{OH})$ vitamin $D$ and zinc levels in patients with acne vulgaris which compared cases and controls in this regard. The mean age of the of acne patients was $20.52 \pm 6.15$ years and in control those treated with supplements was (35.52 \pm 6.14). There were statistical significant differences between group I (case) and group II (control with supplements) in gender male (M): female $(F)$ ratio of ratio of (3:30 versus 18:7) accordingly. As for prevalence, adult acne is more prevalent in women than in men, while in adolescents, the prevalence is quite similar in both sexes. Our results are based on the participant's own perception of the presence or absence of acne rather than a clinical evaluation. Acne continues to be a common skin problem past the teenaged years, with women being affected at higher rates than men in all age groups 20 years or older, although in sex, $\mathrm{BMI}$, and sun exposure hours in a day, there was significant difference when comparing between two groups $(P \leq 0.01)$. However, the prevalence of $25(\mathrm{OH}) \mathrm{D}$ was significantly lower in patients with severe acne compared to supplements administrated group (11.34 vs 35.47; $P \leq 0.001)$. Statistical comparison of acne and control groups revealed that patients 
with acne had no significantly lower serum zinc levels $(P=0.248)$ (Table 1$)$. To understand the vitamin $D$ and levels of zinc status associated with acne patients, we investigated the factors the mean age of the patients who were under 20 years old were defined as having adolescence acne and those over 20 years old as having adult acne, most cases of adult acne are of persistent acne in a population of randomly selected individuals with similar demographic characteristics, the levels of zinc mean according to age of patients with acne lower than 20 years was (83.51 \pm 28.35$)$ while in those over 20 years old the mean was $(78.82 \pm 15.07)$ while in $25(\mathrm{OH}) \mathrm{D}$ was $(11.51 \pm 9.16)$ in adolescence acne and (25.98 \pm 35.72$)$ in adult acne which revealed that patients with acne had highly significantly differences $(P \leq 0.001)$. Statistically variance occur when compared zinc levels according to gender, mean were (77.14 in female and 85.58 in male) with
25(OH)D serum levels (13.88 9.27 in female, $35.61 \pm 47.70$ in male). Although obesity and decreased sun exposure using sunscreen are known to be associated with low 25(OH)D levels, they were not associated with vitamin $D$ and zinc levels deficiency in this study $(P \geq 0.05)$. However, we demonstrate that skin types are not associated with zinc and vitamin D levels which has non-significantly difference according to severe acne patients. Furthermore, we considered family history and onset of acne in male and female adults. Furthermore, we saw that no significant difference was found in mean of family history according to both zinc and vitamin $D$ levels in acne patients $(P \leq 0.001)$. Furthermore, no significant difference was found in mean GAGS score of patients who had exposed to sunlight less than two hours per day and patients with sun-exposure time longer than two hours per day (Table 2).

Table 1: Baseline demographic and clinical characteristics of patients with acne and controls.

\begin{tabular}{|c|c|c|c|}
\hline & Cases I & Control II & $P$-values \\
\hline Age (Years) (M \pm SD) & $20.52 \pm 6.15$ & $35.52 \pm 6.14$ & $\leq 0.001^{* *}$ \\
\hline BMI (M \pm SD) & $23.55 \pm 4.68$ & $26.74 \pm 3.25$ & 0.012 \\
\hline Gender (F:M) (n/\%) & $\begin{array}{c}30(90.9 \%): 3 \\
(9.1 \%)\end{array}$ & $\begin{array}{c}7(28 \%): 18 \\
(72 \%)\end{array}$ & $\leq 0.001^{* *}$ \\
\hline $\begin{array}{l}\text { Sun Exposure hours in a day } \\
\text { ( } M \pm S D)\end{array}$ & $1.32 \pm 1.35$ & $1.70 \pm 1.80$ & 0.315 \\
\hline Vitamin D level (M \pm SD) & $11.34 \pm 7.75$ & $35.47 \pm 43.16$ & $\leq 0.001^{* *}$ \\
\hline 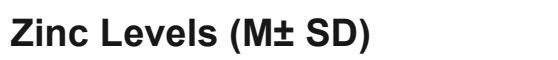 & $73.68 \pm 25.07$ & $83.75 \pm 11.64$ & 0.248 \\
\hline
\end{tabular}


The threshold of $25(\mathrm{OH}) \mathrm{D}$ deficiency in this study was defined as deficient $\leq 12 \mathrm{ng} / \mathrm{ml}$, Adequate $\geq 20 \mathrm{ng} / \mathrm{ml}$ and Inadequate 12_20 $\mathrm{ng} / \mathrm{ml}$ of vitamin $\mathrm{D}$, according to the guideline set by the Food and Nutrition Board of the institute of Medicine. ${ }^{24}$ In total we determined whether vitamin D deficiency, $15 \%$ inadequate levels, $79 \%$ in the severe acne patients were $25(\mathrm{OH})-\mathrm{D}$ deficient, whereas only $6 \%$ adequate levels of vitamin $D$ in acne patients (Figure 1). We also assessed the efficacy of vitamin $D$ deficiency in acne patients $(n=33)$ were randomly assigned in comparison with control group $(n=25)$ which treated with vitamin $D$ supplements for 3 months resulted in a statistically highly significant increase in $25(\mathrm{OH})$ levels $(P \leq 0.001)$ and produced a clinical improvement compared to control group. The acne patients showing zinc level's deficiency where given zinc supplement for 3 months where put in control group, the two group didn't significantly differ by any demographic or clinical factor (Figure 2)

Table 2: Results of vitamin D and Zinc levels according to influencing factors.

\begin{tabular}{lccc}
\hline & Vitamin D & Zinc & $P$-values \\
\hline Age & $11.51 \pm 9.16$ & $83.51 \pm 28.35$ & 0.001 \\
$\leq 20$ & $25.98 \pm 35.72$ & $78.82 \pm 15.07$ & \\
$\geq 20$ & & & $\leq 0.001$ \\
Gender & $13.88 \pm 9.27$ & $77.14 \pm 21.07$ & \\
F & $35.61 \pm 47.70$ & $85.58 \pm 16.28$ & 0.869 \\
M & & & \\
Skin Type & $19.08 \pm 7.53$ & $84.2 \pm 15.73$ & 0.068 \\
Dry & $18.09 \pm 12.77$ & $77.89 \pm 22.6$ & \\
Oily & $34.82 \pm 64.82$ & $83.24 \pm 13.24$ & $\leq 0.001$ \\
Normal & $15.25 \pm 10.26$ & $75.71 \pm 22.21$ & \\
BMI & $24.34 \pm 36.13$ & $82.05 \pm 18.61$ & \\
Normal or Less (<23) & & & \\
Overweight or obese $(\geq 23)$ & $26.84 \pm 36.67$ & $82.88 \pm 14.58$ & \\
Family History & $11.27 \pm 6.29$ & $74.68 \pm 27.18$ & \\
No & & & \\
Yes & $23.42 \pm 34.85$ & $75.17 \pm 18.17$ & \\
Sun Exposure & $15.92 \pm 8.75$ & $97.58 \pm 14.80$ & \\
$<2$ hr sun exposure & & \\
$\geq 2$ hr sun exposure & & & \\
\hline
\end{tabular}

$25(\mathrm{OH}) \mathrm{D}$ values are presented as mean \pm SD. $P$ values are for comparison of vitamin $D$ with Zinc levels. Age, Gender ${ }^{* *}<0.001$

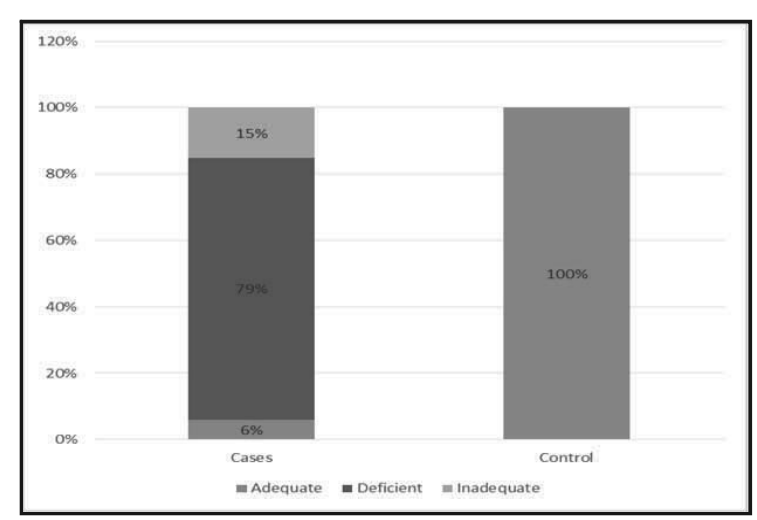

Figure 1: Percentages of patients with different vitamin D levels.

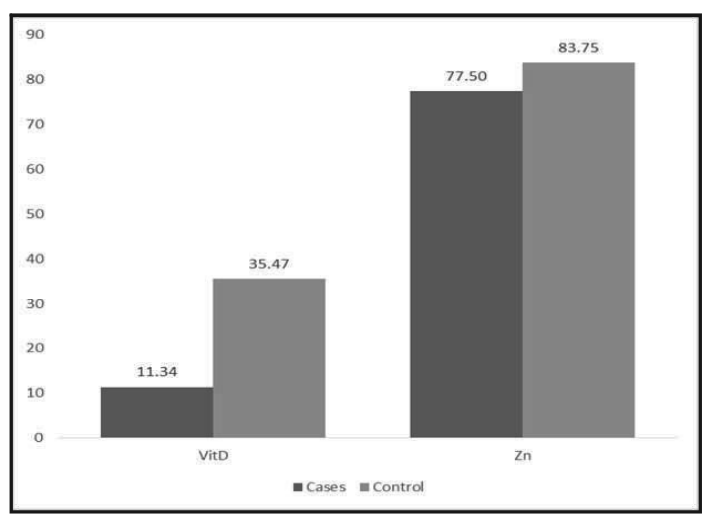

Figure 2: Clinical effects of vitamin $D$ and zinc level in patients with acne in comparison with supplemented control group. 


\section{Discussion}

Acne vulgaris is a chronic inflammatory disease of the pilosebaceous follicles, mainly identified by comedones and inflammatory lesions such as pustules, papules, nodules, cysts, and scars. ${ }^{20}$ This study was carried out in order to determine the levels of vitamin $D$ and zinc levels in acne vulgaris in comparison with those treated with their supplementation therapies on the blood levels. The current study showed that female was more frequent in acne vulgaris, included 33 patients with acne vulgaris (30 females and 3 males) and 25 controls; treated with supplements ( 7 females and 18 males). This was based on the results of previous reports which showed the importance of supplementation therapy which improved the state of acne. ${ }^{21,22}$ However, little is known about the effects of the $25(\mathrm{OH}) \mathrm{D}$ and zinc levels supplementation on the acne vulgaris therapy. In the current study there was statically significant difference in mean age between patients with acne (20.52) and control with supplementation (35.52). An association between age and acne development is unclear, but current research suggests that patients with acne were younger than controls. Several large studies have reported a prevalence of adolescent acne ranging from 81 to $95 \%$ in young men and 79 to $82 \%$ in young women. $^{2}$ Our study found there was no statistically significant association in BMI between patients with acne $\left(23.55 \mathrm{~kg} / \mathrm{m}^{2}\right)$ and control $\left(26.74 \mathrm{~kg} / \mathrm{m}^{2}\right)$ (Table.1). It has also been reported that body fat content is inversely related to serum 25D concentration, and that this associations is stronger than those between 25D and BMI and body weight. ${ }^{23}$ Also found there was no statistically significant association in Sun Exposure hours in a day in acne patients (1.32) and control group (1.70). The main reason for its deficiency is the change of lifestyle, i.e. increase in sunscreen use and environmental factors such as reduced presence sunlight, which is required for ultraviolet (UV) B rays to induce the production of Vitamin $D$ in the skin. ${ }^{24}$ The current study has evaluated the serum concentration of " $25(\mathrm{OH})$ vitamin D" in patients with acne vulgaris (11.34) which significantly increased in control group (35.47) (shown in Table 1). The mechanism of vitamin $D$ functions in some medical conditions has been focused by several researches. Youssef et al (25) as well as other investigators explained an antibiotic effect for vitamin $D$ and its derivatives which help infection prevention too. Zinc, alone or as an adjuvant, has been found useful in many dermatological infections owing to its modulating actions on macrophage and neutrophil functions, natural killer cell/phagocytic activity, and various inflammatory cytokines. ${ }^{16}$ In our study zinc levels presented in Table 2 were no significant difference between acne patients and therapeutic regimen as (73.68) and (83.75), respectively at $P=0.248$. The basis of zinc utilization depends on anti-inflammatory activity. Cytokine production and antioxidant activity are not well known. It can be used in acne treatment based on its anti-inflammatory effects. Zinc levels are evaluated to better understand acne pathogenesis and different results are yielded. ${ }^{26}$ Since patients with severe acne included in the study, there is a strength in our results to document the relationship between the serums zinc levels and the severity of acne (Figure 2). Acne vulgaris can appear at any age but it is more frequent during adolescence, acne vulgaris is more common in males than in females, however in adulthood, acne vulgaris is more common in women than in men. Adolescent acne usually begins with the onset of puberty, when the gonads begin to produce and release more androgens. ${ }^{27}$ As seen in (Table 2) the adolescent acne, males were predominant. In persistent adult acne, males and females were equally affected, while in late-onset adult acne, females were predominant. Hypovitaminosis D is well-documented in those who have had bariatric or gastric 
bypass procedures, in which a malabsorptive state is deliberately induced, ${ }^{28,29}$ but there is no evidence that obesity itself results in reduced absorption of dietary vitamin D. Studies done by vanlint $^{30}$ showing no effect of vitamin $D$ supplementation on weight included participants who were vitamin D replete, and may thus have shown that giving supplemental vitamin $D$ to those who are replete has no additional effect (Table 2). Mechanism to discern body weight and Vitamin D relationship, which include: Vitamin D Receptor (VDR) polymorphism shown in transgenic mice and its overexpression in adipocytes that led to fatty acid $\beta$-oxidation, lipolysis and reduced energy metabolism; increased parathyroid hormone levels in Vitamin D deficiency that can increase adiposity by influx of calcium into adipocytes promoting lipogenesis; Vitamin D as "essential factor" in leptin depletion which may contribute to increased appetite and obesity in Vitamin D deficient conditions; and outdoor activity, food intake and exercise which can also influence Vitamin D levels as confounding factors. ${ }^{31}$ To understand the vitamin D status associated with acne patients, we investigated the factors that influence vitamin $D$ deficiency as well as zinc levels in acne vulgaris patients. In this study, noticed the oily type skin have lower levels of vitamin D and zinc (18.09), (77.89), respectively. It is also noteworthy that skin type determines a person's effectiveness in producing vitamin D. ${ }^{32}$ Serum levels of 25 $(\mathrm{OH}) \mathrm{D}$ in a broad spectrum of different skin diseases and allergic conditions. Although clear definitions of vitamin $D$ insufficiency and deficiency are still lacking, according to the present study we see the highest need to consider vitamin $\mathrm{D}$ supplementation. ${ }^{33}$ Our results suggested that family history of acne was significantly more frequent in subjects with acne (Table 2). Moreover, Di Landroet $a /^{34}$ suggested that acne vulgaris is associated with a family history of acne in first degree relatives, mother and father. Ghodsi ${ }^{35}$ noticed that acne prevalence and severity was influenced the most by mother's acne history. In (Table 2), no significant difference was found in mean GAGS score of patients who had exposed to sunlight less than two hours per day and patients with sun-exposure time longer than two hours per day (Table 2). Although solar exposure is described by several patients as beneficial in controlling acne, it is not known which wave length is responsible for this improvement: UV, visible light, or both. Provitamin D (7dehydrocholesterol, 7-DHC) is converted to previtamin $D$ in the skin by exposure to UVB radiation. The previtamin $D$ is then isomerized by body heat to form vitamin D3. Vitamin D3 is then transported by the blood to the liver, where it is converted to 25-hydroxyvitamin D $\left(25(\mathrm{OH}) D .{ }^{36}\right.$ Exposure to sunlight is having the major role in providing sufficient amount of Vitamin $D$. Everyone should sit in the sun at least for 15 min daily without applying sunscreen so that UV B rays reach to the skin and Vitamin $D$ production will be there. ${ }^{37}$ People who spend their time indoors or wear clothes that cover a large portion of the skin will have more chances of Vitamin $D$ deficiency due to lack of sunlight exposure. Vitamin $D$ is also known by some authorities as a preventive factor against cancers ${ }^{38,39}$ and skin inflammatory diseases like psoriasis, etc. In addition to mediating the beneficial dermatologic effects of light. ${ }^{40}$ In the randomized sample collection of 33 acne patients with vitamin $D$ deficiency, oral vitamin $D$ supplementation in group II of 25 sample produced a significant improvement in acne inflammation (Figure 2). The observed anti-inflammatory effects of vitamin $D$ have several biological mechanisms. The expression of inflammatory biomarkers, such as interleukin (IL)-6, IL-8, and matrix metallo- proteinase 9 , is reduced by treatment with vitamin $D$ in cultured sebocytes. ${ }^{41}$ Our vitamin D supplementation trial had a few potential limitations, such as the use of a low dose and short duration of treatment because 
the risk of hypercalcemia occurs in up to $4 \%$ of the population in association with malignancy, primary hyperparathyroidism, ingestion of excessive calcium and/or vitamin D. ${ }^{42}$ Our study revealed statistically no significantly lower serum zinc levels in patients with acne, as compared with the control group. We believe that the zinc deficiency among the university students participating in this study may be related to inadequate and imbalanced nutrition. The probable association between zinc and acne may be due to the anti-inflammatory action of zinc together with its effects on androgen metabolism and vitamin $A$ metabolism ${ }^{(43)}$. Further studies are required to evaluate the potential effect of zinc gluconate on the acne patients (Figure 2). In conclusion, we found that vitamin $D$ deficiency was more frequent in patients with acne, which was inversely correlated with disease severity, indicating a potential role of vitamin $D$ and zinc deficiency in acne pathogenesis. A further study with a larger sample size is needed to confirm our results because of the small number of patients in the supplementation study and the natural fluctuation of acne. Evaluation of the tissue level of vitamin $D$ in patients with acne will also require a further study to reveal direct evidence of the effect of vitamin $\mathrm{D}$ on acne.

\section{Conclusion}

We found that vitamin $D$ deficiency was more frequent in patients with acne, which was inversely related with disease severity. A further study with a larger sample size is needed to confirm our results because of the small number of patients in the supplementation study and the natural fluctuation of acne.

\section{References}

1. Bolognia JL. Textbook of dermatology. Elsevier. 3th edition 2012; (1):545-59.

2. Burns T. Rook's textbook of dermatology. Blackwell science. 8th edition 2013; 4.

3. Skroza N, Tolino E, Mambrin A, Zuber S, Balduzzi V, Marchesiello A. Adult Acne Versus Adolescent Acne: A Retrospective Study of 1,167 Patients. J Clin Aesthet Dermatol 2018; 11(1):21-25.
4. Dreno B. Treatment of adult female acne: a new challenge. JEADV 2015; 29(5):14-9.

5. Golnick H, Cunliffe W, Berson D. Management of acne: a report from a Global Alliance to Improve Outcomes in Acne. J Am Acad Dermatol 2003; 49:1-38.

6. Tanghetti EA. The role of inflammation in the pathology of acne. J Clin Aesthet Dermatol 2013; 6(9):27- 35.

7. Mohiuddin AK. A Comprehensive Review of Acne Vulgaris. J Clin Pharm 2019; 1(1):17-45.

8. Romańska-Gocka K, Woźniak M, KaczmarekSkamira E, Zegarska B. The possible role of diet in the pathogenesis of adult female acne. Postepy Dermatol Alergol 2016; 33: 416-20.

9. Toossi P, Azizian Z, Yavari H, Fakhim TH, Sadat Amini S, and Enamzade R. Serum 25-hydroxy vitamin $D$ levels in patients with acne vulgaris and its association with disease severity. Clin Cases Miner Bone Metab 2015; 12(3):238-42.

10. FedirkoV, Bostick RM, Long $Q$, Flanders WD, McCullough ML, Sidel- nikov E, Daniel CR, Rutherford RE, Shaukat A. Effects of supplemental vitamin $D$ and calcium on oxidative DNA damage marker in normal colorectal mucosa: a randomized clinical trial. Cancer Epidemiol Bio- markers Prev 2010; 19:280-91.

11. Hamden K, Carreau S, Jamoussi K, Miladi S, Lajmi S, Aloulou D, Ayadi F, Elfeki A. 1Alpha, 25 dihydroxyvitamin D3: therapeutic and preventive effects against oxidative stress, hepatic, pancreatic and renal injury in alloxan-induced diabetes in rats. Nutr Sci Vitaminol 2009; 55:215 -22 .

12. Bilewicz-Stebel1 M, Beata Bergler-Czop B, Sierant K, Wcisło-Dziadecka D. Vitamin D in dermatology. Post N Med 2018; XXXI(1A):55-60.

13. Gupta MA, Gupta AK. The psychological comorbidity in acne. Clin Dermatol 2001; 19:3603 .

14. Birlea SA, Costin GE, Norris DA: Cellular and molecular mechanisms involved in the action of vitamin $D$ analogs targeting vitiligo depigmentation. Curr Drug Targets 2008; 9:34559.

15. Kaymak Y, Adisen E, Erhan M, Celik B, Gurer MA. Zinc Levels in Patients with Acne Vulgaris. J Turk Acad Dermatol 2007;1(3):71302a.

16. Gupta M, Mahajan VK, Mehta KS, and Chauhan PS. Zinc Therapy in Dermatology: A Review. Dermatology Research and Practice 2014; 2014: $1-11$.

17. Olechnowicz J, Tinkov A, Skalny A and Suliburska J. Zinc status is associated with inflammation, oxidative stress, lipid, and glucose metabolism. J Physiol Sci 2018; 68(1):19-31.

18. Nitzan YB and Cohen AD. Zinc in skin pathology and care. J of Dermatological Treatment 2006; 17(4):205-10.

19. Brocard A, Knol A, Khammari A, and Dr'eno B. Hidradenitissuppurativa and zinc: a new 
therapeutic approach-a pilot study. Dermatology 1007; 214(4):325-7.

20. Shen Y, Wang T, Zhou C. Prevalence of acne vulgaris in chinese adolescents and adults. Acta Derm Venereol 2012; 92:40-4.

21. Kramer C, Seltmann H, Seifert M, Tilgen W, Zouboulis CC, Reichrath J. Characterization of the vitamin $D$ endocrine system in human sebocytes in vitro. J Steroid Biochem Mol Biol. 2009;113:9-16.

22. Lim S-K, Ha J-M, Lee Y-H, Lee Y, Seo Y-J, Kim C-D, et al. (2016) Comparison of Vitamin D Levels in Patients with and without Acne: A Case- Control Study Combined with a Randomized Controlled Trial. PLoS ONE 2016; 11(8):e0161162.

23. Arunabh S, Pollack S, Yeh J; Aloia J F. Body fat content and 25-hydroxyvitamin $D$ levels in healthy women. J Clin Endocrinol Metab 2003; 88:15761.

24. Holick MF. Vitamin D deficiency. N Engl J Med 2007; 357:266-81.

25. Youssef DA, Miller CW, El-Abbassi AM, Cutchins DC, Cutchins C, Grant WB, Peiris AN. Antimicrobial implications of vitamin D. Dermatoendocrinol 2011 Oct; 3(4):220-9.

26. Ozuguz P, Kacar SD, Ekiz O, Takci Z, Balta I, and Kalkan G. Evaluation of serum vitamins A and $E$ and zinc levels according to the severity of acne vulgaris. Cutan Ocul Toxicol 2014; 1-4.

27. Raza K, Talwar V, Setia A, Katare O. Acne: An understanding of the disease and its impact on life. International $\mathrm{J}$ of drug development \& research 2012; 4(2):14-20.

28. Cusack C, Danby C, Fallon JC, Ho WL, Murray B, Brady $J$ et al. Photoprotective behavior and sun- screen use: impact on vitamin $D$ levels in cutaneous lupus erythematosus. Photodermatol Photoimmu- nol Photomed 2008; 24:260-7

29. Hewitt S, Søvik T, Aasheim E, Kristinsson J; Jahnsen J, Birketvedt G, et al. Secondary hyperparathyroidism, vitamin $D$ sufficiency, and serum calcium 5 years after gastric bypass and duodenal switch. Obes Surg 2013, 23, 384-90.

30. Vanlint S. Vitamin D and obesity. Nutrients 2013; 5:949-56.

31. Mehmood ZH and Papandreou D. An Updated Mini Review of Vitamin D and Obesity: Adipogenesis and Inflammation State. J of Medical Sciences2016; 4(3):526-532.

32. Mostafa WZ and Hegazy RA. Vitamin D and the skin: Focus on a complex relationship: A review .J of Advanced Research 2015; 6: 793-804

33. Amon U, Baier L, Yaguboglu R, Ennis M, Holick MF, and Amona J. Serum 25-hydroxyvitamin D levels in patients with skin diseases including psoriasis, infections, and atopic dermatitis. Dermatoendocrinol 2018; 10(1):e1442159.

34. Ghodsi SZ, Orawa H, Zouboulis CC. Prevalence, severity, and severity risk factors of acne in high school pupils: a community-based study. J Invest Dermatol2009;129:2136-41.

35. .Di Landro A, Cazzaniga S, Parazzini F. Family history, body mass index, selected dietary factors, menstrual history, and risk of moderate to severe acne in adolescents and young adults. J Am Acad Dermatol 2012; 67:1129-35.

36. Ola Engelsen. The Relationship between Ultraviolet Radiation Exposure and Vitamin D Status. Nutrients 2010; 2:482-95.

37. Wadhwa Sh, Shikha D, Sharma1, Mehta M, Thakur D, Mahajan S, Singh SK, Satija S. Vitamin D deficiency, skin, and sunshine: A review. International $\mathrm{J}$ of Green Pharmacy 2018(Suppl); 12(2):S345.

38. Marashian SM, Farnia P, SeyfSh, Anoosheh S, Velayati AA. Evaluat- ing the role of vitamin $D$ receptor polymorphisms on susceptibility to tuberculosis among Iranian patients: a casecontrol study. Tüberkülozve Toraks Dergisi 2010; 58(2):147-53.

39. Holick MF. Vitamin D: a millenium perspective. J Cell Biochem 2003; 88:296-307.

40. Reichrath J, Lehmann B, Carlberg C, Varani J, Zouboulis CC. Vitamins as hormones. Horm Metab Res 2007 Feb; 39(2):71-84.

41. Lee WJ, Choi YH, Sohn MY, Lee SJ, Kim do W. Expression of Inflammatory Biomarkers from Cultured Sebocytes was Influenced by Treatment with Vitamin D. Indian J Dermatol 2013; 58:327.

42. Tebben PJ, Singh RJ, and Kumar R. Vitamin DMediated Hypercalcemia: Mechanisms, Diagnosis, and Treatment. Endocr Rev 2016; 37(5):521-47.

43. Igic PG, Lee E, Harper W, Roach KW. Toxic effects associated with consumption of zinc. Mayo Clin Proc 2002; 77:713-6. 